\title{
The use of ionic liquids in the processing of chitosan/silk hydrogels for biomedical applications
}

\author{
Simone S. Silva, ${ }^{* a, b}$ Tírcia C. Santos, ${ }^{a, b}$ Mariana T. Cerqueira, ${ }^{a, b}$ Alexandra P. Marques, ${ }^{a, b}$ Lara L. Reys, ${ }^{a, b}$ \\ Tiago H. Silva, ${ }^{a, b}$ Sofia G. Caridade, ${ }^{a, b}$ João F. Mano ${ }^{a, b}$ and Rui L. Reis ${ }^{a, b}$
}

Received 1st December 2011, Accepted 12th March 2012

DOI: 10.1039/c2gc16535j

Natural polymers are adequate renewable resources for the processability of well-defined architectures for several applications. Combinations of polysaccharides and proteins may mimic the naturally occurring environment of certain tissues. The main goal of this work renders the application of green chemistry principles, namely the use of ionic liquids (ILs) and biorenewable sources, such as chitosan (CHT) and silk fibroin (SF), to process new hydrogel-based constructs. Although the solubilization of both materials in ILs has been studied individually, this work reports, for the first time, the role of ILs as solvent, for the production of hydrogels from blends of chitosan and silk fibroin (CSF). These systems offer the advantage of being homogeneous and presenting easy and short dissolution time of both biomacromolecules. Moreover, the use of chitosan obtained from $\alpha$ - and $\beta$-chitin allowed the production of blended hydrogels with distinct physical-chemical properties. In vitro assays demonstrated that these hydrogels supported the adhesion and growth of primary human dermal fibroblasts. Taken these properties together, the CSF hydrogels might be promising biomaterials to be explored for skin tissue engineering approaches.

\section{Introduction}

Natural polymers are adequate renewable resources for the processability of well-defined architectures for several biomedical applications. ${ }^{1}$ Combinations of polysaccharides and proteins may mimic the naturally occurring environment of certain tissues. ${ }^{2}$ In skin tissue engineering, for instance, hydrogels are valuable resources either as dressings for some types of burns and other wounds, or as delivery vehicles for bioactive molecules. ${ }^{3,4}$ The interest in using hydrogels is associated to their ability to absorb high water contents, maintaining a moist wound environment. They can also be easily removed without trauma to the patient. ${ }^{3,4}$ Based on these facts, considerable attention has been focused on hydrogel-based cellular constructs for skin healing. ${ }^{5}$ In that case, critical hydrogel design parameters include both physical properties (such as appropriate mechanical strength and integrity) and biological properties (such as cell behavior, cell-binding domains, enzyme recognition sites). ${ }^{4,6}$ Despite the availability of natural resources, the difficulty in finding common solvents for processing some of the available natural polymers has limited the expansion of their applications,

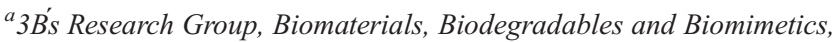
University of Minho, Headquarters of the European Institute of Excellence on Tissue Engineering and Regenerative Medicine, AvePark, 4806-909 Guimarães, Portugal.E-mail: simonesilva@dep.uminho.pt; Fax: +351 253510909; Tel: +351253510900

${ }^{b}$ ICVS/3B's - PT Government Associate Laboratory, Braga/Guimarães, Portugal
}

in particular from biopolymers blends. ${ }^{2}$ Ionic liquids (ILs) appear as alternative solvents and additives which permit combining different families of natural polymers, improving their processability. ${ }^{7,8}$ ILs are commonly defined as organic salts, which are liquids at room temperature. They are composed by an anion and cation that can be chosen within a wide range, allowing the design of a particular IL, tailor made to meet the demands of a certain application. ${ }^{9}$ Moreover, ILs can be foreseen as interesting alternatives to conventional organic solvents, due to their properties, such as good thermal stability, high ionic conductivity, negligible vapor pressure and their easy recovery. ${ }^{7,9}$ Meanwhile, the effective "greenness" of ILs has been evaluated from the points of view of their synthesis, properties and applications. ${ }^{7,9,10}$ Besides, the particular ability of some ILs to work as plasticizers, ${ }^{10}$ catalysts $^{11}$ and solvents, ${ }^{7,12}$ accompanied by a series of concurrent advantages, enables the development of improved processing strategies for the production of biopolymerbased advanced materials. Concerning their application as solvents, most of the studies described in the literature ${ }^{13,14}$ are related to cellulose. However, other works involving chitin, chitosan, cork, silk, and even raw biomass under relatively mild conditions have also been reported. ${ }^{15-17}$ Despite the great potential of ILs as solvents for those aforementioned biomacromolecules and their further application, issues such as biocompatibility and mechanical properties of the developed matrices had not yet been fully explored. Particularly for biomacromolecules such as chitosan and silk fibroin, the use of ILs has been limited to aspects correlated to solubilization and the potential of film- 
forming. ${ }^{13,14,17}$ Chitosan is a deacetylated derivative of chitin, which is isolated from marine resources such as crustacean shells, squid pens and shrimps, ${ }^{2,18}$ by removal of the other components. However, research studies have shown that chitin can be extracted or dissolved directly from crustacean cells using 1ethyl-3-methylimidazolium acetate $([\mathrm{C} 2 \mathrm{~min}] \mathrm{OAc}) .{ }^{19}$ Interestingly, chitosan has been showing high potential in wound healing applications due to its biodegradability, haemostatic activity, anti-microbial and wound healing acceleration properties. $^{2}$ Moreover, the molecular weight and degree of desacetylation (DD), i.e.,. the number of glucosamine units present in the structure of chitosan, have been implicated in many of those properties and consequently, to the biological response of chitosan. ${ }^{20}$ On the other hand, silks are well-known natural fibers produced by a variety of silkworm insects and spiders including Bombyx mori, which is one of the most widely studied sources. $^{21}$ Silk fibroin (SF) is also considered to be a suitable biomaterial for biomedical applications since it possesses good water vapor and oxygen permeability, blood compatibility, and improves collagen deposition and fibroblasts proliferation. ${ }^{21}$ Further works ${ }^{12,17}$ demonstrated the versatility of silk IL solutions and their ability to produce silk fibroin films with a controlled topography.

Blended systems based on silk fibroin and chitosan have been studied, but processed as porous sponges, films and patches for further use as biomaterials in distinct biomedical applications to mimic components of the extracellular matrix (ECM). ${ }^{22,23}$ Despite the application of the chitosan/silk fibroin system using different solvents, ${ }^{22,23}$ the use of ILs in their processing is still not well studied in the literature. Then, the present work focuses mainly on the creation of a new strategy for the production of chitosan/silk based hydrogels to be used as wound dressings. These hydrogels were produced using the green chemistry principles, herein represented by use of ILs, chitosan and silk fibroin. Despite the individual studies of solubilization of chitosan and silk fibroin in ILs, ${ }^{12,17,24}$ this work reports, for the first time, the use of [bmim][Ac] as a common solvent for the formation of CSF/IL blended systems. Furthermore, an evaluation of the effect of chitosan with distinct degree of deacetylation on the production of hydrogels, along with their stability, morphology, mechanical properties and cell behaviour was performed.

\section{Results and discussion}

A facilitated and more efficient dissolution of chitosan and silk fibroin was achieved using an IL, namely 1-butyl-3-methyl imidazolium acetate, [bmim][Ac], as solvent. Some authors ${ }^{25}$ have shown that this IL, is more efficient at disrupting the inter- and intramolecular hydrogen bonding in the biopolymers than $[\mathrm{bmim}][\mathrm{Cl}]$. Thus, [bmim][Ac] was chosen to promote the formation of the chitosan/silk fibroin (CSF)/IL blended systems. It was also found that the CSF/IL blended system offers a variety of new structural and processing possibilities whereas only the hydrogel formation was explored in this work. Comparing the methodologies described in the literature, ${ }^{26-28}$ this approach has some advantages, such as easy dissolution of both biopolymers at high concentrations $(\geq 10 \mathrm{wt} \%$, depending on the polymer) with subsequent formation of hydrogels, less energy demand and is an environmentally friendly process due to the chemical and thermal stability of the solvent and its low vapor pressure. ${ }^{9}$ Besides, the resulting chitosan/silk-based solutions using ILs, have more stability and, can be stored for 1-2 weeks in an inert atmosphere.

Furthermore, the processing of polysaccharides using ILs has the advantages of allowing the formation of hydrogels through dissolution, subsequent cooling induced gelation, and soaking in water or ethanol. This characteristic can be interesting for some polysaccharides, e.g. xantham gum, as they do not readily form hydrogels by usual gelation. Izawa and Kadokawa ${ }^{29}$ described that the dissolution of xantham gum in IL, followed by cooling and gelation promoted the formation of hydrogels with good mechanical properties and thermally induced shape-memory effect.

Besides solvent, the effect of the degree of deacetylation of chitosan on the properties of the developed hydrogels was also evaluated. Depending on the source of chitin (e.g., crab, squid, shrimp), chitin can assume different conformations, namely $\alpha$ and $\beta$, which lead to different properties. ${ }^{30,31}$ The chosen source for $\beta$-chitin was squid pen, which have a high $\beta$-chitin content, whose potential use in biomedical applications has not been completely explored. A comparison with commercial chitosan obtained from $\alpha$-chitin derived from crab was also included. It might be expected that chitosan with different DD would have some influence on the physicochemical and biological properties of the developed hydrogels, as suggested in other works. ${ }^{20,32}$ With this purpose, chitosan with distinct degree of deacetylation was obtained from $\beta$-chitin (CHTS, DD $=91.6 \%$ ), and it was compared to commercial chitosan $(\mathrm{CHTC}, \mathrm{DD}=71.6)$. $\beta$-chitin from squid pens is more susceptible to the deacetylation reaction than $\alpha$-chitin due to its relatively weak intermolecular forces ${ }^{32}$ and, this characteristic can be the explanation for the production of a chitosan with a high DD (91.6\%). The obtained blended solutions were homogeneous, viscous and colorless at a concentration of $4 \mathrm{wt} \%$, which was shown to be the most suitable concentration for hydrogel processing. The CSF solutions were transferred to specific moulds, where low-temperature $\left(4{ }^{\circ} \mathrm{C}\right)$ aging and the immersion of the moulds in ethanol promoted the gelation of the system, and consequently the formation of CSF hydrogels, with very good consistency. Moreover, the resulting CSF solutions have extended stability and easy processability, which allow the moulding of the CSF hydrogels into a range of shapes and sizes and thus, its varied applications.

Although ILs are considered as green solvents, studies have shown that many commonly used ILs exhibit a certain level of toxicity. ${ }^{33}$ As a result, the IL removal from hydrogels is a very important step in the process. In our previous work, ${ }^{16}$ the IL removal process was optimized using different extraction steps in order to produce matrices with very low cytotoxicity. In the present work, the IL removal procedure was first carried out by immersion of the CSF hydrogels in ethanol for 24 hours, followed by Soxhlet extraction with ethanol for 3 days. At the end, the developed CSF hydrogels have a rubbery consistency and soft appearance, as observed in Fig. 1. The findings herein show that the mechanical stability of CSF50C was better than CSF50S, while CSF30S was comparable to CSF50S. 


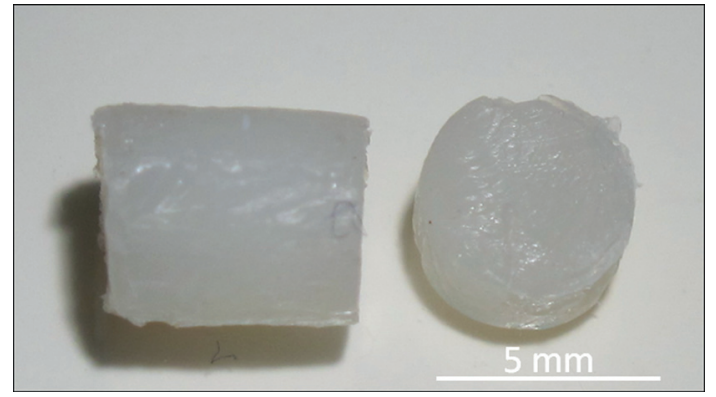

Fig. 1 Representative optical micrographs of the CSF hydrogels obtained after moulding and immersion in ethanol ( 24 hours) at room temperature.

\section{Morphology}

CSF hydrogels were observed by ESEM (Fig. 2). The structure present homogeneous macrostructures characterized by a rough surface, where no phase separation was detected, indicating that the components (chitosan and silk fibroin) have good miscibility between them. Miscibility in a polymer blend is associated with specific interactions between the polymeric components. ${ }^{34}$ In our CSF system, interactions such as hydrogen bonding can be created due to homogeneity of their dissolution in [bmim][Ac]. Also, the micro-roughness of both CSF50C and CSF50S hydrogels (Fig. 2A and 2B) can be associated to high interaction degree between the components, since both were blended in a similar proportion. However, the increase of silk ratio in the CSF30S promoted the formation of a smooth surface (Fig. 2C). Silk hydrogels with similar smooth surfaces were observed by Motta et al., ${ }^{35}$ which appears as its common characteristic.

CSF hydrogels were freeze-dried to obtain sponges, whose morphologies were also investigated by SEM (see Fig. 3). The CSF materials have homogeneous macrostructures. The crosssections of CSF50S and CSF50C (Fig. 3A2-B2) have a lamellar structure similar to observed in chitosan scaffolds, ${ }^{36}$ while different pores sizes appeared in both surface and cross-sections of CSF30S.

\section{Structural characterization}

FTIR analysis was performed to assess the structural changes on the CSF matrices. Fig. 4 shows the FTIR spectra of the CSF matrices with different compositions. As expected the characteristic absorption bands of both silk and chitosan appeared in the proportion to the CSF ratio used. Nevertheless, the displacement of the NH (1519 to $1597 \mathrm{~cm}^{-1}$ ) and $\mathrm{CO}\left(1635\right.$ to $\left.1654 \mathrm{~cm}^{-1}\right)$ for higher wavenumbers suggested the occurrence of conformational changes into these systems, probably as a result of the structural rearrangement of the chains. According to literature the formation of a polyelectrolyte complex system of silk fibroin and chitosan occurs between carboxylate moieties on silk fibroin and protonated amines on chitosan. ${ }^{37}$ Nevertheless, in our case, the mechanism could be different since the dissolution of the biopolymers was performed using [bmim][Ac]. It could be also possible that the disruption of the extensive hydrogen bonding network by [bmim] $[\mathrm{Ac}]$ had occurred with the creation of new strong hydrogen bonding between the anion acetate and
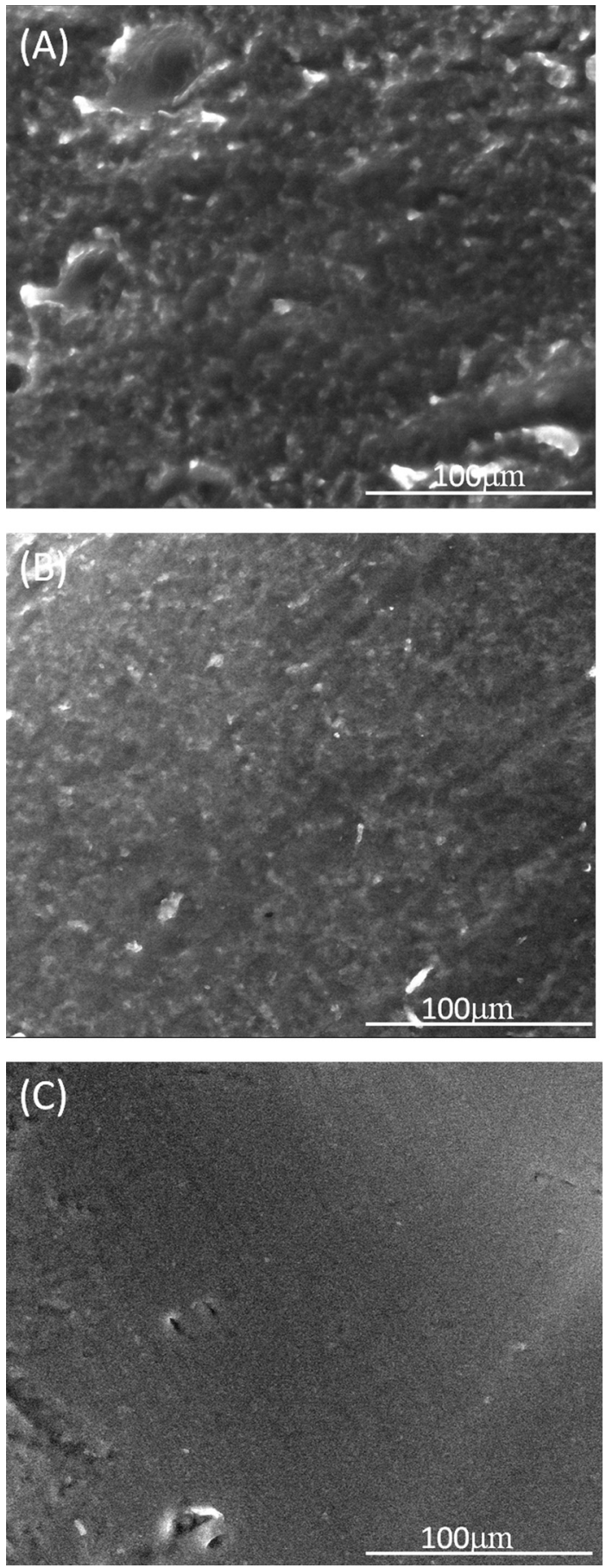

Fig. 2 ESEM images of the surface of the hydrogels; CSF50C (A), CSF50S (B) and CSF30S (C).

hydroxyl groups of the chitosan, as observed in other works. ${ }^{14,19,24}$ Moreover, the CSF30S spectrum (Fig. 4b) showed absorption bands at $1519 \mathrm{~cm}^{-1}$ (amide II) and $1635 \mathrm{~cm}^{-1}$ (amide I), which were attributed to the beta-sheet conformation of silk fibroin, ${ }^{38}$ induced by the treatment of CSF30S hydrogels with methanol.

The XRD profiles of the individual components (CHTC, CHTS and SF after degumming process) and CSF hydrogels are shown in Fig. 5. All diffractograms exhibited few diffraction 

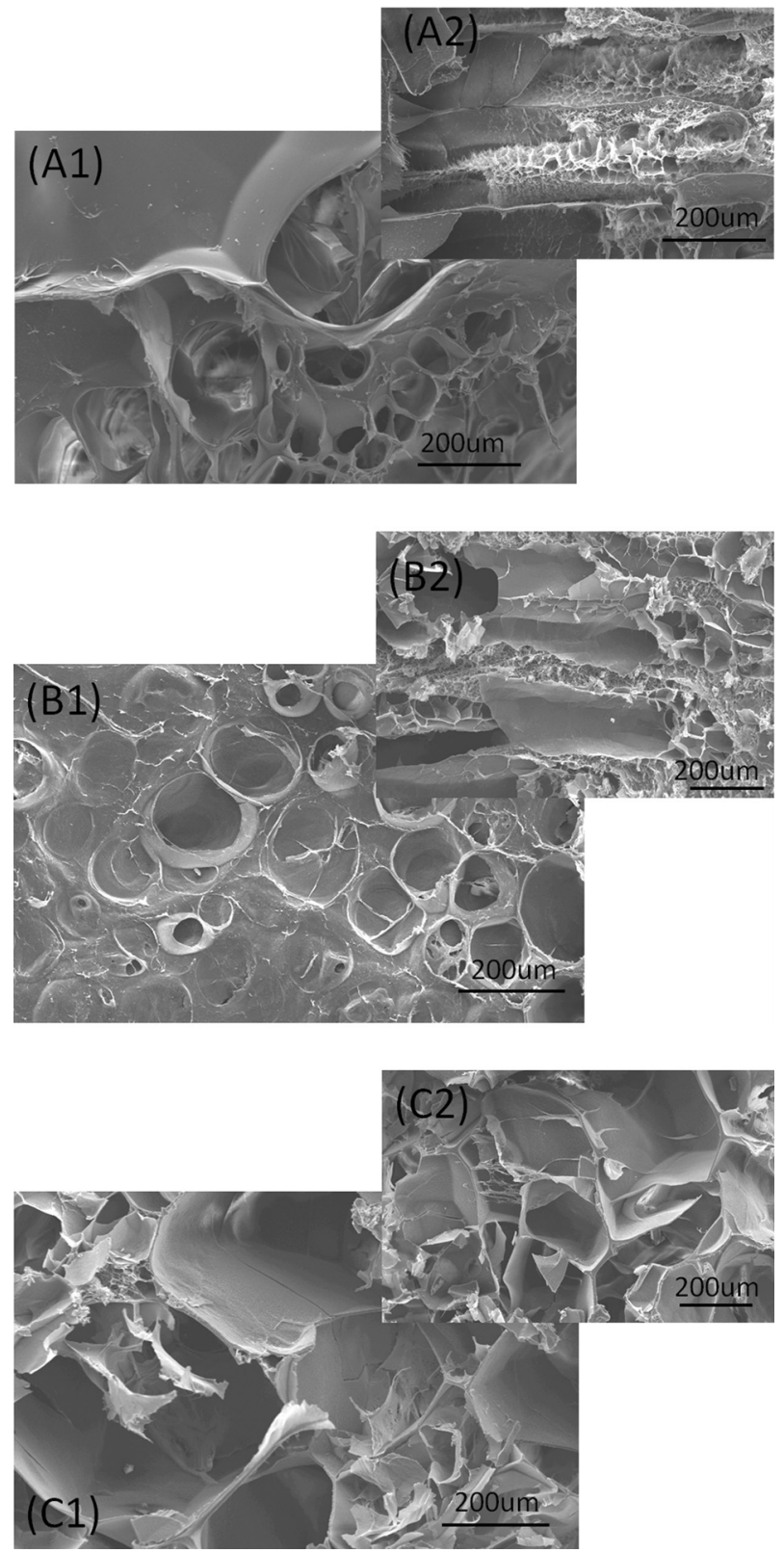

Fig. 3 SEM images of surface and cross-sections of the hydrogels, CSF50C (A), CSF50S (B) and CSF30S (C), where (A1), (B1) and (C1) correspond to their surface and (A2), (B2) and (C2) representing their cross-sections.

peaks. The XRD pattern of both CHTC and CHTS (Fig. 5A) showed broad diffraction peaks at $2 \theta=10^{\circ}$ and $21^{\circ}$, which are typical fingerprints of semi-crystalline chitosan. ${ }^{39}$ In the XRD profile of silk fibroin (Fig. 5A), the presence of a broad amorphous halo indicated that the pure SF, without any treatment, exists in random coil structure. We detected in all XRD profiles of CSF hydrogels (Fig. 5B) the presence of a broad peak at $2 \theta=$ $21^{\circ}$, indicating their amorphous nature. Moreover, the crystallinity values $(18 \%, 12 \%$ and $21 \%$ for CSF50C, CSF 50 S and CSF30S, respectively) corroborate their generally amorphous nature. A possible explanation for these findings might be the breakdown of some hydrogen bonds by [bmim][Ac], followed by a rearrangement of the polymeric chains.

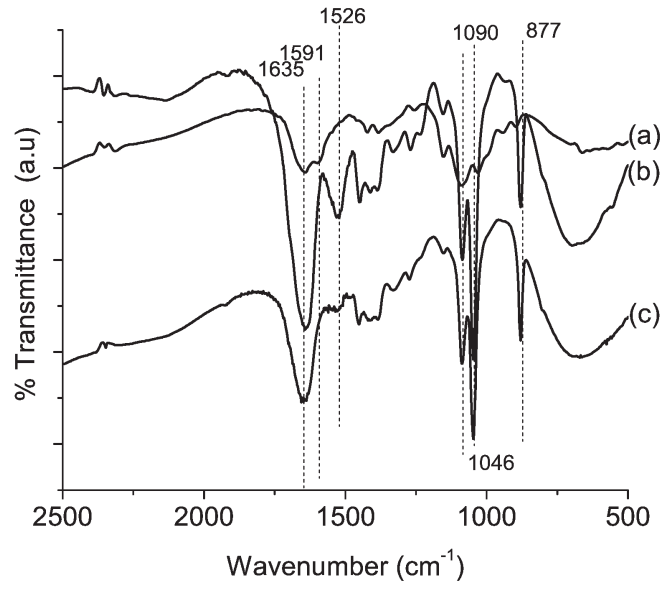

Fig. 4 FTIR spectra of CSF30S (a), CSF50S (b) and CSF50C (c).
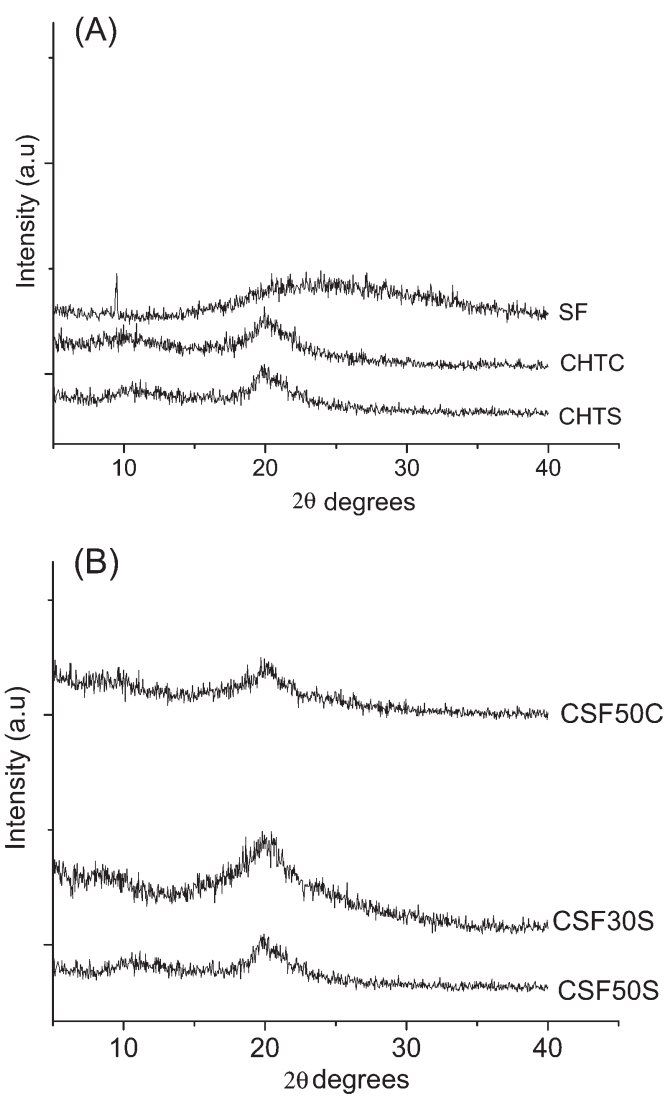

Fig. 5 XRD patterns of (A): SF (after degumming), purified CHTC, purified CHTS; (B): CSF50C, CSF50C and CS30S.

\section{Mechanical and viscoelastic properties}

The viscoelastic characterization of the CSF hydrogels was performed using DMA. This technique gives important indications about the mechanical properties performance that these matrices may have once implanted in vivo. ${ }^{40}$ Human skin tissue is classified as a viscoelastic material; thus, the mechanical response to loading involves both a viscous component associated with energy dissipation and an elastic component associated with energy storage. ${ }^{41}$ The DMA experiments were performed in 

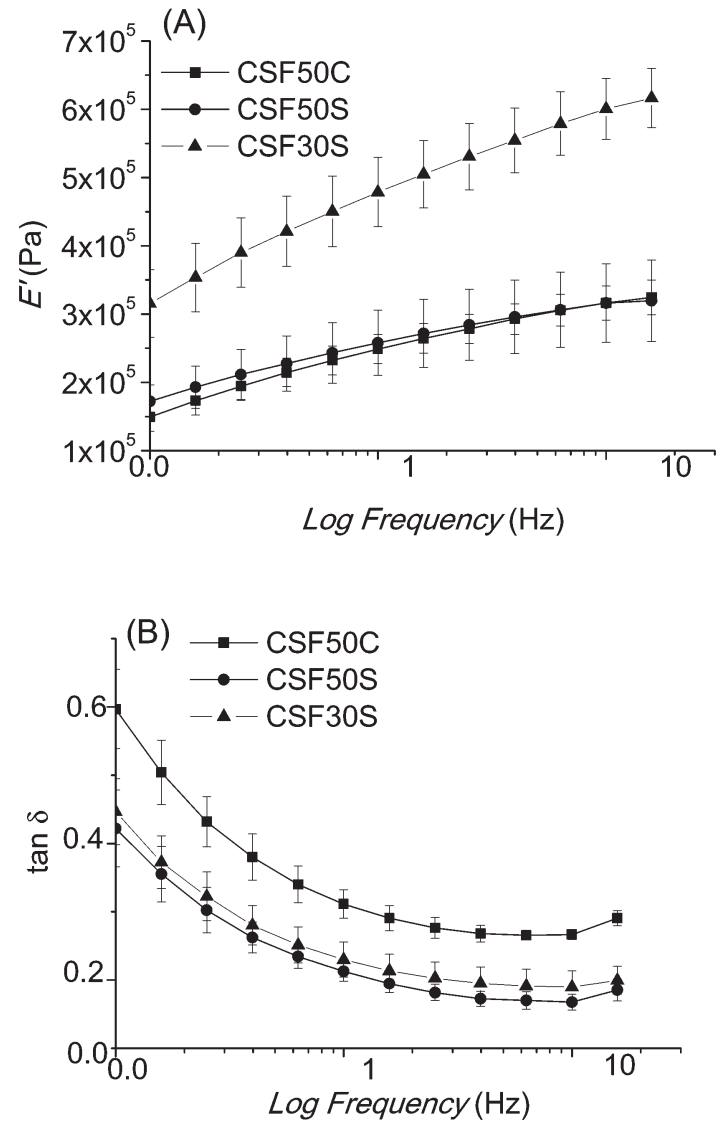

Fig. 6 Evaluation of the viscoelastic properties f CSF hydrogels using DMA; (A) storage modulus $\left(E^{\prime}\right)$ and (B) loss factor $(\tan \delta$ ). Frequency scans were performed in a range of 0.1 to $10 \mathrm{~Hz}$ in wet conditions at $37^{\circ} \mathrm{C}$.

a hydrated environment at $37^{\circ} \mathrm{C}$ to assess how hydrogels behave in more realistic conditions. Fig. 6 presents the viscoelastic behavior of the CSF hydrogels, where storage (elastic) and loss (viscous) components of the complex modulus were determined. The storage modulus $\left(E^{\prime}\right)$ of all CSF tends to increase when increasing the frequency (Fig. 6a). However, there was no significant difference between CSF50C and CSF50S, except when the silk ratio increased (CSF30S). In fact, this improvement in mechanical properties of CSF30S could be attributed to conformational transition from random coil to a $\beta$-sheet structure, as observed in its FTIR spectrum (Fig. 4), which promoted the formation of a rigid network. The influence of the hydrogels composition on the loss factors $(\tan \delta$ ) is presented in Fig. 6b. The loss factor is the ratio of the amount of energy dissipated by viscous mechanisms relative to energy stored in the elastic component, and provides information about the properties of the material. ${ }^{40}$ For all formulations, $\tan \delta$ decreases with the increase of frequency, showing that the materials became more elastic. Values typically higher than 0.2 were observed, evidencing the clear viscoelastic nature of the structures. It was observed that CSF50S and CSF30S presented similar behaviors of $\tan \delta$ which were distinct from the CSF50C. In general, the CSF hydrogels have such mechanical properties in wet conditions that could be suitable for skin healing.

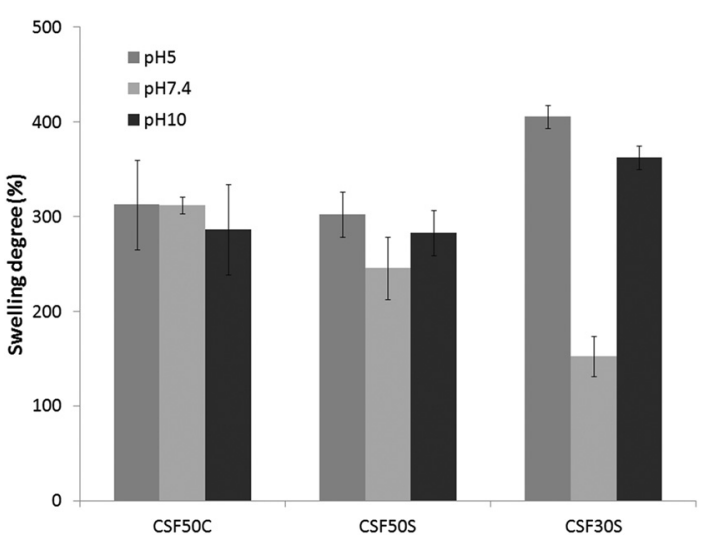

Fig. 7 Comparative swelling ratio of CSF hydrogels after 24 hours of immersion time in different $\mathrm{pHs}$. Data represent the mean \pm standard deviation $\left({ }^{*} p<0.05\right.$, two-way ANOVA).

\section{Swelling studies}

Hydrogels can swell rapidly when subjected to an appropriate environment, meaning specific $\mathrm{pH}$, temperature, electric field, light, and pressure. ${ }^{42}$ The determination of the swelling behavior of a hydrogel is necessary to investigate their applicability in many biomedical applications, such as wound dressings or in drug and protein delivery systems. To study the materials' water uptake abilities and their responses to the external $\mathrm{pH}$ conditions, hydrogels were immersed in buffer solutions at $\mathrm{pH} 5,7.4$ and 10. In Fig. 7, all CSF hydrogels have different swelling degree according to the studied $\mathrm{pHs}$, which were dependent on the composition. In general, the CSF hydrogels showed a high swelling behavior at low $\mathrm{pH}$. According to literature ${ }^{39}$ the swelling of chitosan hydrogels at low $\mathrm{pH}$ can be attributed to a protonation of the amine groups of chitosan. The protonation promotes the solubility of the polymeric segments and leads to polymer chain repulsion, and the interaction with water molecules allow the uptake of more water into the gel network. Moreover, it can be noted that the CSF hydrogels network swells significantly at basic $\mathrm{pH}$ and in neutral conditions. These results could be associated to porosity of the materials, and also the isoelectric point of silk. Statistical higher differences were detected between CSF50C and CSF30S at $\mathrm{pH}$ 7.4. In the overall, the swelling results demonstrated that the developed CSF hydrogels exhibited a $\mathrm{pH}$-dependence pattern that could be explored in specific applications where it is required specific changes in the physical properties of the biomaterial as a response to external stimulus.

\section{Cell behaviour}

One of main purposes of this work was to develop chitosan/silk based hydrogels to be used as wound dressings, and therefore the assessment of the behaviour of skin cells in contact with the materials was pursued. Taking this possible purpose in account, the human dermal fibroblasts (HDFs) were seeded on the CSF hydrogels to verify their adhesion behaviour (SEM images), the metabolic activity (MTS test) and proliferation (DNA quantification). In the SEM images (Fig. 8), high cellular density on the surface of CSF hydrogels was observed. These results suggest that the combination of chitosan and silk fibroin as hydrogels 


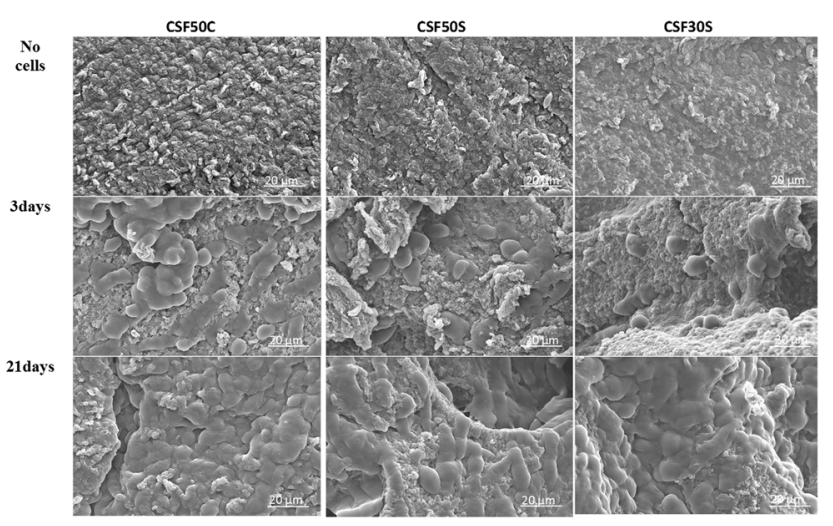

Fig. 8 SEM images of hDFs seeded on CSF hydrogels during 3 and 21 days of the studied culture time period.

provided a good environment for hDFs attachment and growth. An earlier report ${ }^{37}$ showed that silk fibroin/chitosan scaffolds facilitated the growth and attachment of feline fibroblasts, which is in agreement with our results, confirming the potential of this combination. Furthermore, the MTS assay (Fig. 9A) demonstrated the metabolic activity of hDFs during the experimental culture time periods (3, 7, 14 and 21 days) in contact with all CSF hydrogels. Statistical analysis of the data evidenced significant differences $(p<0.05)$ in the metabolic activity at 21 days between CSF50C and both CSF50S and CSF30S. The results of DNA quantification showed that hDFs are able to proliferate from day 3 to day 21 when in culture with the developed CSF hydrogels. Nevertheless, the DNA quantification results (Fig. 9B) demonstrated a higher number of cells in the CSF30S at day 21 which together with the MTS results, seem to indicate that hDFs are less metabolic active on this surface. This might be due to the lack of space to proliferate. Statistical differences were also observed on cell proliferation of hDFs in contact with the CSF50S and CSF50C at 14 days. Additionally, when the silk ratio increased (CSF30S), a substantial increase of cell proliferation along the culture was observed. In fact the number of cells on the CSF30S was significantly higher, at days 14 and 21 days, in comparison to CSF50S and CSF50C. These results clearly suggested the influence of silk ratio on this result. In fact, the introduction of proteins into matrix materials may improve cell behavior as they are able to interact favourably with cells through specific recognition domains. The positive influence of silk fibroin over the attachment and proliferation of both human and animal cell lines has been demonstrated before. ${ }^{21,43}$

The MTS and dsDNA results were consistent with those observed by SEM (Fig. 8), suggesting that hDFs were able to attach and proliferate during the tested culture time. Overall, the performed biological studies suggested that the cells that were seeded onto CSF30S presented better cellular responses when compared with other formulations (CSF50C and CSF50S). Besides, the SEM and DNA data provided an adequate examination of the hydrogel surface, translated in the increase of cell proliferation along the culture time period and therefore confirm that CSF hydrogels are not cytotoxic.

Moreover, these findings also indicated that there was no influence of degree of desacetylation of chitosan on cell behavior of the developed hydrogels.

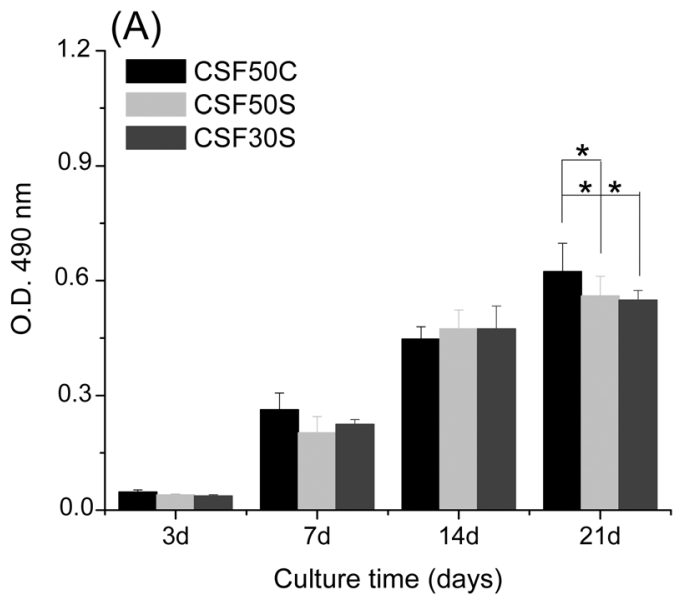

(B)

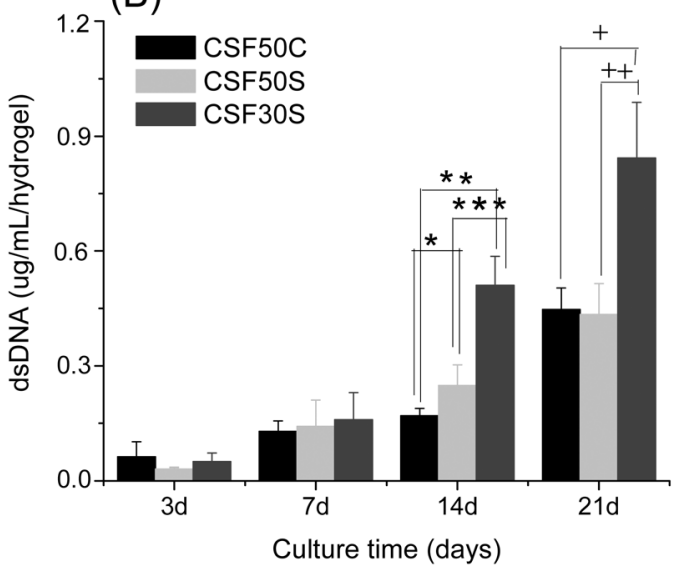

Fig. 9 MTS results (A) and dsDNA content (B) of hDFs cells on CSF hydrogels as a function of culture time. Data represent the mean \pm standard deviation ( $p<0.05$, two-way ANOVA).

\section{Conclusions}

In this work we proposed the processing of hydrogels using the principles of green chemistry involving the use of natural polymers and [bmim] $[\mathrm{Ac}]$ as a common solvent. CSF hydrogels with a soft and rubbery consistency and homogeneous surface were successfully produced. Depending on the composition, the CSF hydrogels have microporous, lamellar structure and viscoelastic behavior. The findings also suggested that the properties of the developed hydrogels were influenced by increased silk ratio, and there was no influence of degree desacetylation of chitosan. Additionally, in vitro biological performances revealed a positive influence over the adhesion and proliferation of $\mathrm{hDFb}$, and demonstrated the potential of CSF hydrogels to sustain dermal fibroblasts adhesion. This work constitutes a strong basis for future healing studies on skin regeneration.

\section{Experimental section}

\section{General information}

Reagent grade medium molecular weight chitosan-CHTC (Sigma Aldrich, CAS 9012-76-4) with a deacetylation degree of 
$76.6 \%$, and molecular weight of $166 \mathrm{kDa}$ by ${ }^{1} \mathrm{H}$ NMR and SEC-MALLS, respectively was used.

Extraction of squid chitin and its conversion in $\beta$-chitosan. Squid chitin was isolated from the endoskeletons of the giant squid Dosidicus Gigas using the methodology described previously. ${ }^{36}$ Briefly, chitin was isolated through a deproteinization step by treating the raw material with $1 \mathrm{M}$ sodium hydroxide $(\mathrm{NaOH})$ solution at $1: 15$ solids to solution ratio at room temperature for 24 hours. After that, squid chitosan (CHTS) was obtained using a deacetylation reaction with $50 \% \mathrm{NaOH}$ during 8 hours. The solution was filtered, and the material was washed with water and dried at room temperature. Both commercial chitosan (CHTC, produced from crab) and CHTS were purified by a re-precipitation method, as described previously. ${ }^{44}$ The degree of deacetylation (DD) and molecular weight (MW) of both CHTS and CHTC were determined by ${ }^{1}$ HNMR and SEC-MALL, respectively. ${ }^{45,46}$ The DD values found were $91.6 \%$ and $76.6 \%$, while MW was $92 \mathrm{kDA}$ and $116 \mathrm{kDA}$ for CHTS and CHTC, respectively. The IL, 1-butyl-3-methyl imidazolium acetate ([bmim][Ac]) from Sigma Aldrich was chosen as solvent and used without further purification. Silk fibroin (SF) from cocoons of Bombyx mori, kindly supplied by the association APPACDM (Castelo Branco, Portugal) was degummed to remove the sericins. The degumming process was achieved by boiling the silk filaments for 1 hour in water containing $1.1 \mathrm{~g} \mathrm{~L}^{-1}$ of $\mathrm{Na}_{2} \mathrm{CO}_{3}$, followed by $30 \mathrm{~min}$ in water with $0.4 \mathrm{~g} \mathrm{~L}^{-1}$ of $\mathrm{Na}_{2} \mathrm{CO}_{3}$. Finally, the resulting fibroin filaments were extensively rinsed in boiling distilled water and air-dried at room temperature (RT).

Characterization. Morphology of the samples was observed using an environmental scanning electron microscope (E-SEM, Quanta 400FEG ESEM), operating in low vacuum mode, and a NanoSEM-FEI Nova 200 equipped with an energy-dispersive spectrometer for silicon microanalysis. Cell morphology and proliferation of hDFbs seeded on CSF hydrogels were also observed by SEM. The infrared spectra were run on a Shimadzu-IR Prestige 21 spectrometer $(\mathrm{KBr})$. X-Ray diffraction (XRD) patterns were collected on a Bruker AXS D8 Discover diffractometer (40 $\mathrm{kV}, 40 \mathrm{~mA}$ ) with Ni-filtered $\mathrm{CuK}$ radiation. Crystalline values were calculated using WinPLOTR software. Dynamic mechanical analysis (DMA) in wet conditions was conducted through compression cycles of increasing frequencies ranging from 0.1 to $10 \mathrm{~Hz}$ using a Tritec 8000B DMA (Triton Technology, Nottingham-shire, United Kingdom). The measurements were carried out at $37{ }^{\circ} \mathrm{C}$ to determine the storage modulus $\left(E^{\prime}\right)$ and loss factor $(\tan \delta$ ). Swelling behaviour of the CSF matrices was determined through their immersion in three different $\mathrm{pH}$ solutions $(\mathrm{pH} \mathrm{5,} \mathrm{pH} 7.4$ and $\mathrm{pH} 10)$ for 24 hours at $37{ }^{\circ} \mathrm{C}$. The swollen samples weight was measured after removing the excess of surface water by gently tapping the surface with a dry piece of filter paper. The degree of swelling for each sample was calculated using equation: $Q\left[\left(W_{\mathrm{s}}-W_{\mathrm{d}}\right) / W_{\mathrm{d}}\right] \times 100$, where $W_{\mathrm{s}}$ is the swollen sample weight under specified environmental conditions and $W_{\mathrm{d}}$ is the dry sample weight. Each experiment was repeated three times, and the average value was considered to be the water uptake value. Statistical analyses of the data (swelling, MTS and DNA) were conducted using two-way ANOVA with Dunnett's post test by using GraphPadPrism version 5.0 for Windows
(GraphPad Software, San Diego, http://www.graphpad.com). Differences between the groups with $p<0.05$ were considered to be statistically significant.

Preparation of the chitosan/silk based hydrogels. For preparing the hydrogels, CHTC, CHTS and SF were dissolved individually in [bmim] [Ac] at $95{ }^{\circ} \mathrm{C}$ with a concentration of the $4 \mathrm{wt}$ $\%$, and then homogeneized in the ratio 50/50 and 30/70 chito$\mathrm{san} /$ silk fibroin. The formulation 30/70 was prepared only from CHTS. After homogeneization, the blended solutions were transferred to specific polystyrene moulds, followed by gelation of the systems through their immersion in ethanol to obtain the chitosan/silk gels. Furthermore, the 30/70 formulation was treated with methanol for $10 \mathrm{~min}$ to stabilize the silk portion. The [bmim][Ac] removal procedure was first carried out by immersing the materials in ethanol for 24 hours, followed by soxhlet extraction with ethanol for 3 days. The identification of the chitosan/silk fibroin (CSF) hydrogels was CSFXY, where X indicated the chitosan content in the blend and $\mathrm{Y}$ the chitosan source (S, squid; C, crab), respectively.

Isolation and culture of human dermal fibroblasts. Primary human dermal fibroblasts (hDFbs) were isolated from adult skin, normally discarded tissue after routine plastic surgery of healthy patients, under collaboration with Hospital da Prelada, Porto, Portugal. The skin was initially cut into small pieces and put in $2.4 \mu \mathrm{mL}^{-1}$ Dispase II (Sigma) overnight, allowing the removal of the epidermal layer. The remaining dermis was further incubated with $0.1 \%$ collagenase type IA (Sigma) at $37{ }^{\circ} \mathrm{C}$ for 3 hours. The resulting suspensions were filtered using a $100 \mu \mathrm{m}$ cell strainer (BD Biosciences), centrifuged and finally the pellet was resuspended in the minimum essential medium (MEM), alpha-medium (Invitrogen), supplemented with 10\% fetal bovine serum and $1 \%$ antibiotic/antimycotic (both gibco, Invitrogen). Medium was changed three times a week and cells were used in passage 6 .

Cell culture. Prior to cell culture studies, the hydrogels were sterilized by autoclave and kept in sterile PBS. In vitro cell tests were performed using a cell suspension of $\mathrm{hDFbs}$ at a concentration of $3.3 \times 10^{4}$ cells per sponge (with $4.2 \mathrm{~mm}$ diameter and $4.6 \mathrm{~mm}$ thickness). Cell-hydrogels constructs were incubated at $37{ }^{\circ} \mathrm{C}$ in a humidified $95 \%$ air and $5 \% \mathrm{CO}_{2}$ atmosphere in MEM alpha-medium (Invitrogen), supplemented with $10 \%$ foetal bovine serum, FBS (Gibco, UK), 2 mM L-Glutamine (Sigma, USA) and antibiotic/antimycotic ( $1 \% \mathrm{~A} / \mathrm{B}$, Gibco, UK) solution. Culture medium was replaced twice a week. Triplicates were used per each time point and per each different test. After 3, 7, 14 and 21 days of culture, the medium was removed and samples were washed with PBS and processed for SEM analysis, MTS test and DNA quantification. MTS (3-(4,5-dimethylthiazol2-yl)-5-(3-carboxymethoxyphenyl)-2-(4-sulfophenyl)- $2 H$-tetrazolium) test was performed to assess the metabolic activity of hDFs cells in contact with CSF hydrogels along the culture. For this assay, an MTS solution was prepared using a 1:5 ratio of MTS reagent and culture medium containing DMEM without phenol red, 10\% FBS and 1\% A/B solution, followed by a 3 hours incubation period at $37{ }^{\circ} \mathrm{C}$. Finally, the optical density (OD) was read at $490 \mathrm{~nm}$, on a multiwell microplate reader 
(Synergy HT, Bio-Tek Instruments). All tests were performed using 6 replicates.

hDFbs proliferation in the CSF hydrogels was determined using a fluorimetric double strand DNA (dsDNA) quantification kit (PicoGreen ${ }^{\circledR}$, Molecular Probes, Invitrogen, UK). Herein, samples collected at 3, 7, 14 and 21 days were transferred into $1.5 \mathrm{ml}$ microtubes containing $1 \mathrm{ml}$ of ultra-pure water. hDFbshydrogel constructs were incubated for 1 hour at $37{ }^{\circ} \mathrm{C}$ in a water-bath and then stored in a $-80{ }^{\circ} \mathrm{C}$ freezer until testing. Prior to dsDNA quantification, constructs were thawed and sonicated for $15 \mathrm{~min}$. Samples and standards (ranging between 0 and $2 \mathrm{mg} \mathrm{ml}^{-1}$ ) were prepared and mixed with a PicoGreen solution in a 200:1 ratio, and placed on an white 96 well plate. Triplicates were made for each sample or standard. The plate was incubated for $10 \mathrm{~min}$ in the dark and fluorescence was measured on a microplate reader (BioTek, USA) using an excitation and emission wavelengths of $485 / 20 \mathrm{~nm}$ and $528 / 20 \mathrm{~nm}$, respectively. A standard curve was created and sample dsDNA values were read off from the standard curve graph.

\section{Acknowledgements}

S. S. Silva, T. H. Silva and S. G. Caridade thank the Portuguese Foundation for Science and Technology (FCT) for post-doctoral and $\mathrm{PhD}$ fellowships (SFRH/BPD/45307/2008, SFRH/BPD/ $34704 / 2007$ and SFRH/BD/64601/2009, respectively). This work was partially supported by the FCT (Project PTDC/QUI/ 68804/2006) and FEDER through POCTEP 0330 IBEROMARE_1_P.

\section{Notes and references}

1 J. F. Mano, G. A. Silva, H. S. Azevedo, P. B. Malafaya, R. A. Sousa, S. S. Silva, L. F. Boesel, J. M. Oliveira, T. C. Santos, A. P. Marques, N. M. Neves and R. L. Reis, J. R. Soc. Interface, 2007, 4, 999-1030.

2 S. S. Silva, J. F. Mano and R. L. Reis, Crit. Rev. Biotechnol., 2010, 30, 200-221.

3 S. Huang and X. Fu, J. Controlled Release, 2010, 142, 149-159.

4 S. Van Vlierberghe, P. Dubruel and E. Schacht, Biomacromolecules, 2011, 12, 1387-1408.

5 S. C. Rizzi, Z. Upton, K. Bott and T. R. Dargaville, Expert Rev. Med. Devices, 2010, 7, 143-154.

6 K. Y. Lee and D. J. Mooney, Chem. Rev., 2001, 101, 1869-1880.

7 T. Ueki and M. Watanabe, Macromolecules, 2008, 41, 3739-3749.

8 O. A. El Seoud, A. Koschella, L. C. Fidale, S. Dorn and T. Heinze, Biomacromolecules, 2007, 8, 2629-2647.

9 J. Anthony, J. Brennecke, J. Holbrey, E. Maginn, R. Mantz, P. Trulove, A. Visser and T. Welton, in Ionic Liquids in Synthesis, ed. P. Wasserscheid and T. Welton, Wiley-VCH, Weinheim, 2002, pp. 41-55.

10 H. Zhao, Chem. Eng. Commun., 2006, 193, 1660-1677.

11 H. Olivier-Bourbigou, L. Magna and D. Morvan, Appl. Catal., A, 2010, $373,1-56$.
12 M. K. Gupta, S. K. Khokhar, D. M. Phillips, L. A. Sowards, L. F. Drummy, M. P. Kadakia and R. R. Naik, Langmuir, 2007, 23, 13151319.

13 J. Vitz, T. Erdmenger, C. Haensch and U. S. Schubert, Green Chem., 2009, 11, 417-424.

14 A. Pinkert, K. N. Marsh, S. S. Pang and M. P. Staiger, Chem. Rev., 2009, 109, 6712-6728.

15 T. Q. Yuan, J. He, F. Xu and R. C. Sun, Prog. Chem., 2010, 22, 472-481.

16 S. S. Silva, A. R. C. Duarte, A. P. Carvalho, J. F. Mano and R. L. Reis, Acta Biomater, 2011, 7, 1166-1172.

17 D. M. Phillips, L. F. Drummy, D. G. Conrady, D. M. Fox, R. R. Naik, M. O. Stone, P. C. Trulove, H. C. De Long and R. A. Mantz, J. Am. Chem. Soc., 2004, 126, 14350-14351.

18 N. Alves and J. Mano, Int. J. Biol. Macromol., 2008, 43, 401-414.

19 Y. Qin, X. M. Lu, N. Sun and R. D. Rogers, Green Chem., 2010, 12, 968-971.

20 Y. Yuan, B. Chesnutt, W. Haggard and J. Bumgardner, Materials, 2011, 4, 1399-1426.

21 C. Vepari and D. L. Kaplan, Prog. Polym. Sci., 2007, 32, 991-1007.

22 S. S. Silva, A. Motta, M. T. Rodrigues, A. F. M. Pinheiro, M. E. Gomes, J. F. Mano, R. L. Reis and C. Migliaresi, Biomacromolecules, 2008, 9, 2764-2774.

23 Z. X. Cai, X. M. Mo, K. H. Zhang, L. P. Fan, A. L. Yin, C. L. He and H. S. Wang, Int. J. Mol. Sci., 2010, 11, 3529-3539.

24 H. Xie, S. Zhang and S. Li, Green Chem., 2006, 8, 630-633.

25 N. Sun, H. Rodriguez, M. Rahman and R. D. Rogers, Chem. Commun., 2011, 47, 1405-1421.

26 D. Huemmerich, U. Slotta and T. Scheibel, Appl. Phys. A: Mater. Sci. Process., 2006, 82, 219-222.

27 C. Zhao, J. Yao, H. Masuda, R. Kishore and T. Asakura, Biopolymers, 2003, 69, 253-259.

28 C. Zhong, A. Kapetanovic, Y. Deng and M. Rolandi, Adv. Mater., 2011, 23, 4776-4781.

29 H. Izawa and J.-I. Kadokawa, J. Mater. Chem., 2010, 20, 5235-5241.

30 M. Rinaudo, Prog. Polym. Sci., 2006, 31, 603-632.

31 M. Rhazi, J. Desbrières, A. Tolaimate, A. Alagui and P. Vottero, Polym. Int., 2000, 49, 337-344.

32 K. Kurita, Mar. Biotechnol., 2006, 8, 203-226.

33 D. Zhao, Y. Liao and Z. Zhang, Clean, 2007, 35, 42-48.

34 S. S. Silva, B. J. Goodfellow, J. Benesch, J. Rocha, J. F. Mano and R. L. Reis, Carbohydr. Polym., 2007, 70, 25-31.

35 A. Motta, C. Migliaresi, F. Faccioni, P. Torricelli, M. Fini and R. Giardino, J. Biomater. Sci., Polym. Ed., 2004, 15, 851-864.

36 L. Reys, S. Silva, J. Oliveira, S. Caridade, J. Mano, T. Silva and R. Reis, J. Bioactive Compat. Polym., 2012, under revision.

37 N. Bhardwaj and S. Kundu, Carbohydr. Polym., 2011, 85, 325-333.

38 M. Tsukada, Y. Gotoh, M. Nagura, N. Minoura, N. Kasai and G. Freddi, J. Polym. Sci., Part B: Polym. Phys., 1994, 32, 961-968.

39 C. Bangyekan, D. Aht-Ong and K. Srikulkit, Carbohydr. Polym., 2006, 63, 61-71.

40 J. F. Mano, R. L. Reis and A. M. Cunha, in Polymer Based Systems on Tissue Engineering, Replacement and Regeneration, ed. R. L. Reis and D. Cohn, Kluwer, Dordrecht, 2002, vol. 86, pp. 139-164.

41 F. Silver, J. Freeman and D. DeVore, Skin Res. Technol., 2001, 7, 18-23.

42 N. A. Peppas and P. Colombo, J. Controlled Release, 1997, 45, 35-40.

43 Y. F. Zhang, W. Fan, Z. C. Ma, C. T. Wu, W. Fang, G. Liu and Y. Xiao, Acta Biomater. 2010, 6, 3021-3028.

44 R. Signini and S. P. C. Filho, Polím. Ciência Tecnol., 2001, 11, 58-64.

45 A. Hirai, H. Odani and A. Nakajima, Polym. Bull., 1991, 26, 87-94.

46 S. Nguyen, S. Hisiger, M. Jolicoeur, F. M. Winnik and M. D. Buschmann, Carbohydr. Polym., 2009, 75, 636-645. 